Les ANNALES Les Annales de droit

DE DROIT

$7 \mid 2013$

Varia

\title{
Les Observations sur la Coutume de Normandie : vers une unification aux inspirations normandes
}

The Observations sur la Coutume de Normandie: Norman inspiration towards unification

Jérôme Pigeon

\section{(2) OpenEdition}

Journals

Édition électronique

URL : http://journals.openedition.org/add/838

DOI : $10.4000 /$ add. 838

ISSN : 2606-1988

Éditeur

Presses universitaires de Rouen et du Havre

Édition imprimée

Date de publication : 1 avril 2015

Pagination : 177-201

ISSN : 1955-0855

Référence électronique

Jérôme Pigeon, "Les Observations sur la Coutume de Normandie : vers une unification aux inspirations normandes », Les Annales de droit [En ligne], 7 | 2013, mis en ligne le 03 mai 2018, consulté le 30 avril 2019. URL : http://journals.openedition.org/add/838 ; DOI : 10.4000/add.838 


\title{
Les Observations sur la Coutume de Normandie: vers une unification aux inspirations normandes
}

\author{
Jérôme PIGEON
}

Réunir la France «sous l'empire des mêmes lois, comme elle est réunie sous l'autorité du même prince ${ }^{1}$ ", tel est le dessein proclamé en 1771 devant le parlement de Paris, par le chancelier Maupeou. Loin d'être une idée neuve, cette aspiration à un droit commun français s'inscrit dans un mouvement initié depuis le $\mathrm{XvI}^{\mathrm{e}}$ siècle sous l'impulsion de juristes français comme Charles Dumoulin qui, dès 1546, émettait l'idée de réunir toutes les coutumes du royaume en une seule ${ }^{2}$. Après lui et jusqu'à la fin du XVIII ${ }^{\mathrm{e}}$ siècle, d'autres auteurs, à l'image de Coquille, Loysel ou encore Bourjon et Pothier se font les chantres d'un droit commun coutumier ayant le plus souvent pour socle la coutume réformée de Paris ${ }^{3}$. De même, la création en 1679 de professeurs de droit français dans les facultés de droit conduit ces enseignants à comparer diverses coutumes du royaume afin d'en faire ressortir des principes généraux applicables dans l'ensemble du royaume ${ }^{4}$. Les arrêtistes eux-mêmes participent, à travers leurs recueils, à dégager un droit commun ${ }^{5}$. Pour autant, ce mouvement n'est pas unanime et certains nient l'existence d'un droit commun coutumier ${ }^{6}$ ou s'évertuent à défendre la pureté du droit de leur province ${ }^{7}$.

1. Jules Flammermont, Remontrances du parlement de Paris au XVIII siècle, Paris, Imprimerie nationale, 1895 , t. II, p. 188.

2. Jean-Louis Thireau, Introduction historique au droit, Paris, Flammarion, 2009, p. 249.

3. Jean-Louis Halpérin, L'Impossible Code civil, Paris, PUF, 1992, p. 30-32.

4. Ibid., p. 32.

5. Guillaume Leyte, «Le droit commun de la France. Observations sur l'apport des arrêtistes ", Droits, $\mathrm{n}^{\circ} 38,2003$, p. 60-63.

6. Jean-Louis Halpérin, op. cit., p. 34-35. Voir aussi Jacques Krynen, «Le droit romain "droit commun de la France" ", Droits, n ${ }^{\circ}$ 38, 2003, p. 21-35.

7. Guillaume Leyte, art. cité, p. 57. Sur le scepticisme relatif à cette idée d'unification voir Xavier Martin, Mythologie du Code Napoléon: aux soubassements de la France moderne, Bouère, Dominique Martin Morin, 2003, p. 96 et suiv. 
Pour être effective, cette unité du droit nécessitait une volonté politique. Seul le monarque pouvait accomplir cette œuvre par voie législative et, dès la fin du $\mathrm{Xv}^{\mathrm{e}}$ siècle, Louis XI semblait bien avoir conçu un tel projet. Par la suite, ce désir d'unité anima encore le pouvoir royal, mais le droit privé ne relevant traditionnellement pas du domaine de la loi, ses interventions en matière coutumières furent, jusqu'au $\mathrm{XvIII}^{\mathrm{e}}$ siècle, relativement rares et empreintes de prudence ${ }^{8}$. Cependant, l'idée d'unification n'en demeurait pas moins présente et l'œuvre de Colbert dans la seconde moitié du XVII ${ }^{\mathrm{e}}$ siècle en atteste. Certes, si ses ordonnances n'abordent pas véritablement le droit privé, elles montrent les progrès de cette idée d'unité et l'on sait que le contrôleur général des finances envisageait plus largement de parvenir à l'unification de l'ensemble du droit français ${ }^{9}$. Plus encore: sous le règne de Louis XV, cette volonté prit corps au travers des ordonnances du chancelier Daguesseau, ces dernières abordant de véritables matières de droit privé. Mais, là encore, si les progrès sont indéniables, le champ disciplinaire restreint de ces ordonnances révèle que Daguesseau, conscient de la difficulté de la tâche, n'envisageait encore cette unification que de manière progressive et estimait préférable de procéder par étapes afin de ne pas froisser trop brutalement les particularismes provinciaux ${ }^{10}$.

Après la mort de Daguesseau, les réalisations concrètes en la matière subirent un coup d'arrêt. Cependant, la volonté d'unification du droit coutumier était toujours présente et, vingt ans après, le chancelier Maupeou affirmait ouvertement qu'il entendait contribuer à cette entreprise. Robert Villers a montré ${ }^{11}$ que le chancelier n'en était pas resté au stade des simples paroles mais qu'il avait amorcé un véritable travail de fond, exprimé dans les Observations sur la Coutume de Normandie ${ }^{12}$. Ces Observations prennent place dans les pièces justificatives jointes au Compte rendu adressé par Maupeou au roi Louis XVI, en 1789. Dans ce compte rendu, Maupeou abordait de nouveau la question du droit coutumier et réaffirmait qu'ayant eu en charge la justice du royaume,

\footnotetext{
8. Jean-Louis Thireau, op. cit., p. 253-258; Jean-Louis Halpérin, op. cit., p. 37-38.

9. Ibid., p. 39-40.

10. Ibid., p. 40-42.

11. Robert Villers, «Un projet de réforme de la Coutume de Normandie sous le ministère Maupeou ", Revue historique de droit français et étranger (RHD), 1956, p. 356-375.

12. Ces Observations, publiées dans l'article de Robert Villers aux p. 360-375 se trouvent également à la Bibliothèque nationale de France (BNF), fonds français, ms. 6570, consultables désormais sous forme de microfilm à la cote mf 11219 .
} 
il avait effectivement travaillé à l'unification des coutumes ${ }^{13}$. Au-delà des raisons qui l'avaient poussé à accomplir ce travail ${ }^{14}$, il évoquait la méthode suivie pour y parvenir. Comme celle de Daguesseau dans la première moitié du XVIII ${ }^{\mathrm{e}}$ siècle, celle-ci révèle que Maupeou n'entendait pas parvenir à une unification brusque, mais qu'il souhaitait au contraire procéder de manière progressive, et qu'il s'agissait d'un travail de longue haleine. Ainsi, son projet tendait, dans une première phase, à la simplification de ce droit coutumier et à réduire les coutumes à leurs différences et exceptions, puis dans une seconde phase, à former de ces coutumes « une coutume mère à laquelle on aurait tâché de ramener successivement toutes les autres ${ }^{15}$ ".

Seule manifestation concrète de cette initiative, les Observations sur la Coutume de Normandie, rédigées autour de l'année 1770 par CharlesFrançois Lebrun ${ }^{16}$, peuvent laisser à penser qu'une réforme du droit coutumier normand apparaissait comme une priorité à Maupeou et à son entourage. En effet, la coutume de Normandie, parmi les premières à s'être cristallisée ${ }^{17}$, ne fut officiellement rédigée qu'en $1583^{18}$, et à l'inverse de nombreuses autres coutumes, elle n'avait, au XVIII ${ }^{\mathrm{e}}$ siècle, fait l'objet d'aucune réformation ${ }^{19}$. Cette rédaction officielle tardive aurait pu être l'occasion d'une véritable modernisation du droit normand intégrant l'évolution du droit coutumier du royaume, mais au contraire, ses rédacteurs manifestèrent un attachement particulier au droit existant,

13. «J'avais conçu le projet de retrancher de toutes les coutumes les dispositions communes à toutes que j'aurais réunies dans le code général de la France...» (BNF, ms. 6570).

14. Dans son compte rendu, Maupeou dénonçait notamment "leur masse énorme ", «leur style vieilli et inintelligible» (ibid.)

15. Ibid.

16. Robert Villers, art. cité, p. 360.

17. Pour une vue d'ensemble sur la cristallisation des coutumes, voir notamment Jean Bart, Histoire du droit privé de la chute de l'Empire romain au XIx ${ }^{e}$ siècle, Paris, Montchrestien, 2009, p. 97-99; Jean-Louis Thireau, op. cit., p. 124-127. Pour la Normandie, consulter Jean Yver, "Les caractères originaux de la coutume de Normandie ", Mémoires de l'Académie nationale des sciences, arts et belles-lettres de Caen, 1952, t. II, p. 310-314.

18. La coutume de Normandie avait bien été rédigée dès la première moitié du XIII ${ }^{\mathrm{e}}$ siècle, mais la question de l'origine officielle ou privée de cette rédaction pose encore question aujourd'hui. Sur ce point, voir Virginie Lemonnier-Lesage, «Les coutumiers de Normandie: une rédaction sous influence?", Passé et présent du droit, $\mathrm{n}^{\mathrm{o}} 4$, 2007, p. 255-270.

19. Sur la rédaction et la réformation des coutumes, voir notamment Philippe Sueur, Histoire du droit public français, Paris, PUF, 2001, t. II, p. 39-51. 
et firent preuve d'un immobilisme certain ${ }^{20}$. Par la suite, et jusqu'au XVIII ${ }^{\mathrm{e}}$ siècle, cet attachement, sinon de l'ensemble des normands, du moins des juristes de cette province, ne se démentira pas, comme en témoignent l'opinion des différents commentateurs de la coutume ou encore les remontrances du parlement de Rouen relatives à l'ordonnance de Daguesseau sur les donations. Dans celles-ci, les magistrats normands exhortaient le roi à ne pas porter "atteinte aux lois municipales de la province " dans la mesure où la coutume était "dans cet état de perfection qui lui a mérité le titre glorieux de sage coutume " et que, "rédigée avec la dernière exactitude ", elle était parvenue "au point qu'on a plus rien à désirer pour elle que sa conservation ${ }^{21} »$.

Ainsi, le chemin semblait encore long avant que les juristes normands parussent en mesure d'accepter une quelconque idée de réformation de leur droit. Partant de ce constat, il nous a paru opportun d'analyser ces Observations sur la Coutume de Normandie, afin de voir comment Charles-François Lebrun entendait procéder pour parvenir à concilier le vou d'unification avec les résistances de ces juristes dans un contexte politique des plus tendus, où l'opposition entre le pouvoir royal et les parlements du royaume était à son paroxysme. Pour ce faire, nous avons confronté le texte des Observations aux ouvrages de doctrine normands à sa disposition à l'époque. Cette analyse a révélé que les Observations s'intégraient pleinement dans la première phase du projet Maupeou et

20. Cette tendance au conservatisme s'explique notamment par l'origine normande des commissaires nommés par le roi. Jacqueline Musset relève qu'ils étaient tous «d'authentiques normands» et qu'ils "constituaient une chance pour tous ceux qui voulaient conserver leur droit pluriséculaire» («Le parlement et la coutume de Normandie ", dans Du parlement de Normandie à la cour d'appel de Rouen (1499-1999), Paris, Imprimerie nationale, 1999, p. 124-126); voir encore Jean Yver, selon lequel l'un des facteurs de conservation «se trouve dans le caractère authentiquement normand des commissaires qui ont été nommés par le roi pour présider à la rédaction» ("La rédaction officielle de la coutume de Normandie (Rouen 1583). Son esprit », Annales de Normandie, $\mathrm{n}^{\circ}$ 1, 1986, p. 3-36).

21. Archives départementales de Seine-Maritime, registres secrets, 1 B 250. Dans ces mêmes remontrances, les magistrats normands affirmaient que «la plus légère atteinte à cette loy municipale jette les coeurs dans la plus profonde consternation ». Ils exprimaient également leur réserve quant à l'idée d'une unification du droit coutumier: "Sire, l'uniformité de loix est un projet aussy difficile dans le vaste Royaume soumis à V. M. que seroit celuy de réunir tous les esprits des juges dans une unanime conformité de décisions; chaque pays sous les loix qui luy sont propres joüit d'une parfaiste et précieuse tranquilité; elles tendent toutes ces différentes loix par des routes différentes à faire rendre à tous les peuples un homage uniforme au souverain elles mérittent donc la protection de ce même souverain dont elles implorent l'autorité pour leur conservation et leur maintient...» 
que, si elles tendaient, dans une certaine mesure, vers une unification du droit, elles constituaient surtout une œuvre prudente, largement inspirée des réflexions et des aspirations des juristes normands et par-là étaient destinées à faciliter un rapprochement ultérieur plus important du droit normand avec le droit commun coutumier.

\section{Un projet de réforme nourri des aspirations normandes}

Les Observations sur la Coutume de Normandie expriment, à diverses reprises, la nécessité de simplifier et de moderniser le texte de 1583. De ce point de vue, s'il est certain que ce projet répondait aux vœux manifestés par Maupeou, il ressort également des modifications proposées, tant dans la forme que sur le fond, que l'auteur s'est largement inspiré des réflexions de la doctrine et de la jurisprudence normandes.

\subsection{Une nécessaire simplification formelle}

Dans la forme, ce projet entendait apporter davantage de clarté et d'unité à la coutume de Normandie. En cela, s'il était en parfaite adéquation avec la volonté de Maupeou, qui, dans son Compte rendu, avait envisagé de réduire les coutumes «à l'expression la plus simple, la plus vulgaire et la moins équivoque » et de les renfermer dans quelques pages afin de faciliter le travail des jurisconsultes ${ }^{22}$, il répondait encore à une tendance plus large qui sous l'influence de l'École du droit naturel aspirait à des règles de droit simples et claires ${ }^{23}$. Mais, surtout, une telle orientation lui permettait d'aller dans le sens de certaines revendications des juristes normands dont il avait sans nul doute connaissance, ainsi qu'en attestent ses références à la "commune opinion", à certains commentateurs et à la jurisprudence du parlement de Rouen.

Ainsi, l'auteur des Observations préconisait, afin d'apporter de la clarté au texte, d'intervenir d'une manière générale ${ }^{24}$ sur les termes usités, sans cependant pointer précisément tel ou tel terme. Une réécriture dans un langage plus usuel était, selon lui, de nature à éviter, pour l'avenir, un certain nombre de difficultés ${ }^{25}$. Or ses lectures de la doctrine normande n'avaient pu que l'encourager en ce sens. En effet, même si dans leur

22. BNF, ms. 6570.

23. Jean-Louis Halpérin, op. cit., p. 60.

24. Il prenait cependant la peine de préciser, lorsqu'il abordait le titre des retraits, que le terme "clameur» usité notamment dans l'article 454 était un terme normand signifiant retrait.

25. «Lors de cette rédaction les articles qui peuvent renfermer quelqu'ambiguïté seront rédigés en termes plus clairs et propres à prévenir toute difficulté » (BNF, ms. 6570). 
très grande majorité, les auteurs normands considéraient leur coutume comme la plus sage du royaume ${ }^{26}$, un certain nombre n'en admettait pas moins, depuis fort longtemps, la nécessité de moderniser le vocabulaire du texte de 1583. Au début du XVII ${ }^{\mathrm{e}}$ siècle, Josias Bérault reconnaissait, dans son avant-propos au lecteur, avoir été confronté lors de la lecture de la coutume à des «nuages et obscurités ${ }^{27}$ ». Cette réflexion ne lui était pas propre et elle se fit encore plus précise au début du XVIII ${ }^{\mathrm{e}}$ siècle sous la plume de Pierre Merville. En 1707, ce dernier, dans la préface de son ouvrage La Coutume de Normandie réduite en maximes selon le sens littéral et l'esprit de chaque article, était encore plus explicite et virulent. Selon lui, son ouvrage lèverait "toutes les obscuritez que la barbarie du Texte» avait répandues sur «la plus sage Coûtume du Royaume». Cet avocat au parlement précisait sa pensée en ces termes: "Il faut convenir que le texte de cette Coûtume est rempli de termes étrangers et inconnus dans la langue Françoise, et que presque chaque terme a besoin d'explication, sans quoi l'on demeure court, et l'on est arrêté dans la lecture de chaque article ${ }^{28}$.» Il concluait en affirmant que le lecteur, après la lecture de son ouvrage, verrait la coutume de Normandie comme « une Loy qui auparavant étoit embarassée par des termes barbares ${ }^{29}$ ".

La tendance de l'auteur à reprendre, dans son projet, les réflexions de certains auteurs normands ne se cantonnait pas au seul aspect linguistique. Afin de rendre plus intelligible la coutume, il préconisait, en outre, une certaine restructuration de celle-ci. Cette volonté ressort dans plusieurs passages et est particulièrement prégnante lorsque l'auteur aborde le régime matrimonial. Il envisageait de regrouper toute cette

26. Ainsi Roupnel de Chenilly, dans l'épître dédicatoire de la $4{ }^{e}$ édition de l'ouvrage de Pesnelle, écrivait: «La Coutume de Normandie doit principalement sa gloire à la sagesse et à la beauté de ses maximes: toujours occupée utilement du soin de maintenir le lustre des familles, ou d'en entretenir l'harmonie, ses décisions prennent un empire sur tout lecteur judicieux» (Coutume de Normandie expliquée par M. Pesnelle avec les obervations de M. Roupnel de Chenilly, Rouen, R. Lallemant, 1771 , t. I).

27. Josias Bérault, La Coustume réformée du pays et duché de Normandie, anciens ressorts et enclaves d'iceluy, Rouen, 1612.

28. Pierre de Merville, La Coutume de Normandie réduite en maximes selon le sens littéral, et l'esprit de chaque article, Paris, $\mathrm{H}$. Charpentier, 1707, préface.

29. Ibid. David Hoüard, dont le dictionnaire paraîtra après la rédaction de ce projet de réforme, convenait également du caractère parfois obscur de la coutume: «Le texte est-il conçu obscurément? J'examine si l'obscurité vient de ce que les expressions employées par les réformateurs ont vieilli, ou de ce que les mœurs anciennes nous sont peu connues» (Dictionnaire analytique, historique, étymologique, critique et interprétatif de la coutume de Normandie, Rouen, Le Boucher jeune, 1780, t. I, p. XXXIV). 
matière dans un seul titre intitulé Des droits appartenant à gens mariés et au survivant des conjoints et des contrats de mariages. À cette occasion, il précisait que la coutume de Normandie ne contenait pas un pareil titre et qu'il s'agissait de regrouper dans ce dernier des articles qui y étaient relatifs et qui, jusqu'alors, étaient éparpillés dans la coutume ${ }^{30}$. Une telle démarche aurait pour conséquence d'insérer dans ce titre des articles auparavant dispersés dans le titre des successions collatérales, dans celui consacré au douaire, ou encore dans le titre traitant du partage d'héritage. Le régime matrimonial n'était pas le seul objet de cette démarche; toujours dans ce même souci "de clarté et de simplicité ${ }^{11}$ ", il entendait fusionner les titres relatifs aux successions aux propres et au partage d'héritage.

Sur ce point, il semble qu'il y ait eu, là encore, un lien entre les propositions de l'auteur et les aspirations des auteurs normands. En effet, même si à l'époque où ces Observations furent rédigées la nécessité d'une telle réorganisation du corps de la coutume de Normandie n'avait jamais été exprimée aussi clairement par les jurisconsultes normands, ni même par la jurisprudence, il n'en reste pas moins que, dès la première moitié du $\mathrm{XVIII}^{\mathrm{e}}$ siècle, les réflexions de certains auteurs laissent apparaitre qu'une inclination en ce sens s'était fait jour et qu'il y avait un intérêt certain, pour la compréhension de la coutume, à revenir sur l'ordre du texte de 1583. Cette idée est notamment présente chez Charles Routier. Ce dernier s'écartait, dans son ouvrage Principes généraux du droit civil et coutumier de la province de Normandie, du plan de la coutume qui, jusqu'à présent, avait été suivi par les auteurs normands. Sans conteste influencé par la tendance générale, dans le royaume, à suivre le plan des Institutes de Justinien, Routier divisait son ouvrage en trois titres: «Des personnes», «Des biens», « Des actions ${ }^{32} »$. Comme il l'indiquait

30. «La coutume ne contient pas un pareil titre, il serait composé de différents articles épars dans différents titres de la coutume» (BNF, ms. 6570).

31. «La coutume outre le titre des successions aux propres tant en ligne directe que collatérale contient encore un titre intitulé de partage d'héritage; il serait à propos de ne faire qu'un titre des deux, pour plus de clarté et de simplicité » (BNF, ms. 6570).

32. Constatant la difficulté de l'étude du droit, il estimait nécessaire la réalisation d'ouvrages permettant d'en faciliter l'accès et indiquait: "Justinien en sentit la nécessité de son tems; c'est ce qui produisit la composition des institutes qui facilitent l'étude des Loix... Les plus habiles Jurisconsultes, et les plus grands Maîtres, ont fait en France la même chose... Cet ouvrage tend à la même fin... Je l'ai divisé, comme Justinien, en trois parties, qui font les trois objets du Droit; sçavoir, les Personnes, les Choses et les Actions " (Charles Routier, Principes généraux du droit civil et coutumier de la province de Normandie, Rouen, Le Boucher, 1748). 
dans la préface de son ouvrage, cette méthode était de nature à faciliter "l'étude et l'intelligence de notre droit, et servira à en graver plus profondément les principes dans l'esprit ${ }^{33}$ ». De même, l'ouvrage de Jean-Baptiste Flaust, bien qu'écrit près d'une dizaine d'années après la rédaction des Observations visant à réformer la coutume de Normandie, est également révélateur de l'évolution de la doctrine quant à la forme du texte de cette dernière. En effet, dans son Explication de la Coutume et de la jurisprudence dans un ordre clair et facile de 1781 (Rouen, chez l'auteur), cet avocat adoptait une logique similaire à celle ébauchée dans le projet de réforme. L'auteur regrettait l'obligation faite par la coutume à ses lecteurs, et donc aux juristes, de rechercher à de multiples endroits du texte les éléments permettant de connaître les règles régissant un même domaine. Il avouait ainsi que ce caractère épars des articles de la coutume traitant d'une même matière avait été l'une des raisons pour lesquelles il avait entrepris la rédaction d'un tel ouvrage. Il l'indiquait en ces termes dès l'introduction: "L'étude de notre Coutume et de notre jurisprudence est difficile : cela tient [...] de ce que la Coutume a beaucoup de dispositions relatives les unes aux autres, qui sont répandues et dispersées sous des titres différents...» Ainsi, à l'image de ce que projetait l'auteur des Observations sur la Coutume de Normandie, Flaust estimait qu'il convenait, afin d'en faciliter l'étude, de rapprocher « sous des titres convenables, les articles de la coutume et des règlements qui ont de la connexité et des rapports ${ }^{34}$ ».

À partir de ces quelques observations, il apparaît certain que la modernisation formelle de la coutume de Normandie envisagée par l'auteur s'inspirait des réflexions et répondait à une attente des juristes normands. Sur le fond, le constat est identique. Les modifications projetées traduisaient, pour partie, la prise en compte des aspirations normandes et consacraient, dans une certaine mesure, les critiques émises par la doctrine et la jurisprudence.

\subsection{La consécration des critiques}

Au-delà de la forme, l'essentiel du projet portait sur le fond et en ce domaine, le souci de l'auteur de satisfaire les réflexions normandes est encore plus marquant. Les juristes normands, on l'a dit, avaient tous une haute opinion de leur coutume. Cependant, cela ne les empêchait pas d'exprimer, sur certains points, leur incompréhension, voire leur

33. Ibid.

34. Jean-Baptiste Flaust, op. cit., p. 1. 
désaccord avec certaines solutions retenues par les rédacteurs de 1583 . Or il est frappant de voir que, dans son projet, l'auteur des Observations reprenait à son compte, et bien souvent mot pour mot, les positions de la jurisprudence et de la doctrine normande en invoquant la nécessité d'adapter la coutume à l'évolution doctrinale et jurisprudentielle de la province. Ses propos relatifs au titre VIII de la coutume, traitant du bénéfice d'inventaire, sont sur ce point particulièrement significatifs. Dans ce titre, il dénonçait notamment la teneur de l'article 90 de la coutume suivant lequel «avant l'adjudication s'il se présente aucun du lignage du défunt, qui se veuille porter héritier absolu, il y sera reçû, encore qu'il soit plus éloigné que l'héritier par bénéfice d'inventaire, en payant les frais faits par celui qui s'est porté héritier par bénéfice d'inventaire ${ }^{35}$ ». En la matière, nombreuses étaient les coutumes, parmi lesquelles la coutume réformée de Paris ${ }^{36}$, établissant une distinction entre succession en ligne directe et succession collatérale. Dans ces coutumes, l'exclusion de l'héritier bénéficiaire ne valait que dans le cadre d'une succession collatérale et non dans celui d'une succession en ligne directe. Or la généralité des termes de l'article 90 laissait supposer qu'il écartait tout héritier bénéficiaire dès lors qu’un héritier absolu se manifestait.

À l'exception de Bérault qui, au début du XvII ${ }^{\mathrm{e}}$ siècle, semblait adhérer à la teneur de l'article 90 de la coutume en estimant que l'héritier simple faisait honneur au défunt, alors que l'héritier bénéficiaire au contraire lui faisait injure ${ }^{37}$, les commentateurs qui lui succédèrent furent d'avis unanimement contraire. Basnage estimait ainsi : "Nos mauvais praticiens s'étant persuadez qu'il étoit de l'honneur du défunt et de l'interest des créanciers, d'avoir un héritier pur et simple, ils ont été dans cette erreur que l'héritier absolut étoit toûjours préférable à

35. Charles Bourdot de Richebourg, Nouveau Coutumier général, ou corps des coutumes générales et particulières de France, et des provinces connues sous le nom des Gaules, Paris, Brunet, 1724, t. IV, p. 64.

36. Article 342 de la coutume réformée de Paris: «L'héritier en ligne directe, qui se porte héritier par bénéfice d'inventaire, n'est exclus par autre parent qui se porte héritier simple» (Ibid., t. III, p. 54).

37. Josias Bérault, op. cit., p. 117: «L'héritier absolut exclud l’héritier par bénéfice d'inventaire pour deux raisons principales, l'une est la faveur des créanciers, qui se peuvent prendre aux biens de l'héritier absolut aussi bien qu'à ceux du défunt, lesquels sont par l'addition de l'hérédité meslez et confus en un; l'autre est l'honneur du défunt que luy fait l'héritier simple, au lieu que l'héritier par bénéfice d'inventaire luy fait injure.» 
l'héritier bénéficiaire ${ }^{38}$.» Selon eux, lorsque Justinien avait introduit le principe du bénéfice d'inventaire, il avait eu pour unique fin d'empêcher que l'héritier fût chargé des dettes du défunt au-delà des fonds de la succession. Dès lors, ils dénonçaient l'interprétation des anciens praticiens suivant laquelle tant en ligne directe qu'en ligne collatérale, l'héritier simple excluait l'héritier bénéficiaire ${ }^{39}$. Afin de justifier leur position, ils s'appuyaient sur la jurisprudence du parlement de Rouen qui par un arrêt du 7 mars 1662 avait jugé qu'en ligne directe l'héritier bénéficiaire n'était pas exclu par l'héritier pur et simple ${ }^{40}$. Ils citaient également d'autres coutumes du royaume qui avaient adopté la même solution, notamment celles de Paris, Troyes et Orléans ${ }^{41}$.

Dans son projet, force est de constater que non seulement l'auteur des Observations reprenait à son compte la critique de la généralité des termes de cet article, mais qu'il utilisait également les arguments avancés par la doctrine et l'évolution de la jurisprudence normande. Ainsi, il affirmait que les dispositions de l'article 90 de la coutume de Normandie étaient fondées sur le vieux préjugé suivant lequel « il était plus honorable pour la mémoire du défunt que la succession fut prise purement et simplement » et ajoutait que la jurisprudence avait fait évoluer les choses vers davantage de justice ${ }^{42}$.

Cette inclination de l'auteur se retrouve encore quant aux autres modifications à apporter à ce titre VIII. En matière de bénéfice d'inventaire, la convergence de la doctrine et de la jurisprudence normande avec les Observations ne se cantonnait pas au seul article 90. En effet, les commentateurs de la coutume de Normandie considéraient tous que les formalités imposées par la coutume à ceux qui désiraient se porter héritiers par bénéfice d'inventaire étaient excessives. Pour Basnage, «la longueur de ces formalitez apporte aux créanciers et aux héritiers plus de dommage que de profit ${ }^{43}$ ». Il affirmait même que ces formalités imposées à l'héritier étaient superflues et entraînaient des dépenses inutiles.

38. Henri Basnage, Euvres (Commentaire de la Coutume du pays et duché de Normandie et traité des hypothèques), Rouen, Maurry, 1709, t. I, p. 131.

39. Ibid., p. 131 et 134 .

40. Basnage sous l'article 90 indiquait: "C'est aujourd'hui une jurisprudence certaine, que l'héritier bénéficiaire en ligne directe ne peut être privé de la succession par celui qui se porte héritier pur et simple, suivant l'arrêt du 7 mars $1662[\ldots]$ ce qui a été confirmé par plusieurs autres arrêts» (op. cit., p. 134).

41. Ibid.

42. «La coutume ne faisait point la distinction entre les successions directes et collatérales » (BNF, ms. 6570).

43. Henri Basnage, op. cit., p. 131. 
Selon lui, tout cela résultait de la mauvaise compréhension du principe même du bénéfice d'inventaire ${ }^{44}$ et il en arrivait à l'idée que l'ensemble des articles contenus dans le titre relatif au bénéfice d'inventaire aurait pu être réduit au nombre de trois, à savoir les articles 92, 93 et $98^{45}$. Roupnel de Chenilly affirmait quant à lui que « cette partie de notre droit a besoin d'une réformation ${ }^{46} »$. Prenant en compte, sans aucun doute, l'opinion de la doctrine, le parlement de Normandie s'éloigna également du texte de la coutume. Depuis un arrêt du 6 juillet 1729, rendu en faveur d'un nommé Marouard, il avait en effet assoupli ces formalités et exempté de faire les contumaces pour prendre une succession par bénéfice d'inventaire en ligne directe.

L'auteur des Observations soulignait, lui aussi, la lourdeur de ces formalités, le décalage existant entre le texte de la coutume de 1583 , la teneur de la doctrine et l'évolution de la jurisprudence normande ${ }^{47}$. Afin de moderniser le texte de la coutume et de mettre le droit en accord avec la jurisprudence et la doctrine, il reprenait à son compte l'opinion des juristes normands et en arrivait à la conclusion que « la justice et la raison » devaient amener la suppression de six des treize articles du titre consacré au bénéfice d'inventaire et qu'il serait bon d'étendre l'évolution de la jurisprudence aux successions collatérales ${ }^{48}$.

44. Ibid. On retrouve des idées similaires dans l'ouvrage de Pesnelles. Roupnel de Chenilly, qui y apporta ses observations, notait que les coutumes avaient totalement défiguré le bénéfice d'inventaire, l'avaient embarrassé de trop de formalités et en avaient tiré des conséquences absurdes, (op. cit., t. I, p. 98). Voir également Leroyer de La Tournerie, Nouveau Commentaire portatif de la Coutume de Normandie, Rouen, 1778 , t. I, p. 133-134.

45. Henri Basnage, op. cit., p. 131: «Ainsi tous les Articles de ce Titre pouvoient être réduits à trois seulement, dont le premier seroit le XCII, suivant lequel l'héritier bénéficiaire est tenu de faire bon et loial inventaire de tous les biens, titres et enseignemens de ladite succession; le XCIII qui leur ordonne de faire apprécier les meubles; et le dernier de ce titre, par lequel il est tenu de répondre aux actions et demandes des créanciers. »

46. Pesnelle, op. cit., p. 98. Pour sa part, Jean-Baptiste Flaust convenait que les formalités imposées étaient embarrassantes mais ajoutait qu'il fallait cependant s'y conformer (op. cit., t. I, p. 25).

47. «La jurisprudence a établi qu'un descendant héritier présomptif pouvait prendre la succession de son ascendant par bénéfice d'inventaire, en obtenant seulement des lettres à la chancellerie et en demandant seulement l'enthérinement par une simple requête sans être obligé de faire donner d'assignation aux héritiers en général » (BNF, ms. 6570).

48. «La raison et la justice se réunissent pour que la même règle soit observée en succession collatérale et conséquemment que lesdits articles 86 et compris 91 soient entièrement supprimés " (ibid.). Cette idée d'extension aux successions collatérales se retrouvera, en 1778, dans le Nouveau Commentaire portatif de la Coutume de 
Ses propos sur l'article $365^{49}$ de la coutume de Normandie sont tout aussi révélateurs de cette volonté de prendre en compte l'opinion des juristes normands. Selon cet article, lorsque la femme se portait héritière de son mari et donc prenait part aux conquêts, cette part n'était pas affectée par la reprise de la dot dès lors que cette dernière avait été consignée ${ }^{50}$. Cette reprise devait être supportée par la part des conquêts revenant aux héritiers du mari ${ }^{51}$. Cette disposition de la coutume, qui constituait un avantage indéniable pour la femme $e^{52}$, fut ouvertement dénoncée par les commentateurs normands. Parmi eux, Basnage se montra sans aucun doute le plus virulent. Il estimait que les rédacteurs de 1583 s'étaient largement écartés des principes, établis ailleurs dans la coutume, visant à réduire le droit des femmes et à ne pas les enrichir de la dépouille de leur mari. L'article 365 lui apparaissait en tout point contraire à ce principe et on ne peut plus favorable aux femmes ${ }^{53}$. Il ajoutait qu'en dépit de l'absence de communauté entre époux posée par la coutume, l'article 365 constituait comme une incohérence dans la mesure où il conférait aux femmes des avantages dont elles ne seraient pas susceptibles de bénéficier dans les lieux où la communauté avait cours ${ }^{54}$. Dès lors, Basnage exprimait clairement son désir de voir cet article réformé, la distinction que ce dernier établissait entre dot consignée et non consignée ne reposant sur « rien de solide » et cet article ne pouvant servir «qu’à faire fraude à la loi qui défend au mari de faire

Normandie de Le Royer de La Tournerie (op. cit.) Évoquant l'arrêt du parlement de Normandie du 6 juillet 1729, l'auteur estimait : «Il seroit à désirer que la Cour voulut se déterminer à adopter cette jurisprudence, dans le cas de successions collatérales » (op. cit., p. 134).

49. Article 365 : «Femme prenant part aux conquests faits par son mary constant le mariage, demeure néanmoins entière à demander son dot sur les autres biens de son mary, au cas qu'il y ait consignation actuelle du dot faite sur les biens du mary, et où il n'y aura point de consignation, le dot sera pris sur les meubles de la succession, et s'ils ne sont suffisans, sur les conquests » (Charles Bourdot de Richebourg, op. cit., p. 78 ).

50. Voir notamment David Hoüard, op. cit., p. 644: «Il y a dot consignée, et dot qui n'est pas consignée; car quand par le contrat la dot a été actuellement consignée sur les biens du mari, elle prend, s'il meurt, part à ses conquêts, sans que cela l'empêche de réclamer sa dot.»

51. Voir Virginie Lemonnier-Lesage, Le Statut de la femme mariée dans la Normandie coutumière. Droit et pratique dans la généralité de Rouen, Presses universitaires de la faculté de droit de Clermont-Ferrand, 2005, p. 335-338.

52. Ibid., p. 337.

53. Henri Basnage, op. cit., p. 546.

54. Ibid.: «Nous ne souffrons point la communauté, mais cet article leur donne des avantages qu'elles ne pouroient pas obtenir en vertu de la communauté. » 
avantage à sa femme ${ }^{55}$ ». Cette critique sera reprise par les successeurs de Basnage, notamment par Pesnelle:

Les effets de cette consignation [...] répugnent à l'intention de la Coutume, et à la raison: la Coutume a privé les femmes des avantages qui viennent en conséquence de la communauté, et d'ailleurs elle a voulu conserver les propres dans les familles [...] Ces deux principes sont renversés par les effets de la consignation : car en conséquence d'icelle, la femme a plus d'avantages qu'elle ne pourroit avoir par la communauté, vû qu'elle reprend sa dot sur les biens propres de son mari, et que ce nonobstant elle la reprend une seconde fois $^{56}$.

Une nouvelle fois, toutes ces critiques parurent suffisamment convaincantes à l'auteur des Observations sur la Coutume de Normandie pour qu'il les utilisât afin de justifier la réforme de cet article 365. Tout comme les commentateurs, il insistait sur le fait que, par cet article, la femme prenait deux fois la même choses, que cette disposition allait à l'encontre des autres dispositions de la coutume tendant à empêcher le mari de faire des avantages à sa femme; et il notait que «tous les jurisconsultes de la province ont jusqu'à présent senti l'injustice d'une pareille disposition ». Il concluait ses observations en ces termes: «Est-il tolérable qu'une loi renferme des dispositions aussi révoltantes et aussi injustes? Il est à souhaiter que le roi par sa sagesse réforme un pareil abus ${ }^{57}$.»

Enfin, on peut se demander si la suppression envisagée, par l'auteur, du titre XII de la coutume de 1583 relatif aux successions dans le bailliage de $\mathrm{Caux}^{58}$, peut, elle aussi, au-delà de la volonté simplificatrice annoncée ${ }^{59}$, être appréhendée comme la concrétisation des aspirations des juristes normands? Certes, leurs critiques furent moins unanimes

55. Ibid., p. 547.

56. Pesnelle, op. cit., t. I, p. 402. L'auteur poursuivait en ces termes: «Toutes ces conséquences sont contraires à la raison, qui ne veut pas que les femmes s'enrichissent des dépouilles de leurs maris, en se faisant payer deux fois d'une même chose; et d'ailleurs n'ont pour fondement que le mot consignation; plus propre à fournir la matière d'une vaine imagination, que celle d'aucun raisonnement solide. » L'annotation de Roupnel de Chenilly, sous ce même article, allait dans le même sens puisqu'il affirmait que la consignation était un terme imaginaire et se demandait: «Pourquoi lui avoir donné le pouvoir de renverser les principes les plus constants de notre jurisprudence, et d'enlever quelquefois les trois quarts de la succession de son mari?»

57. BNF, ms. 6570.

58. Sur la succession dans le bailliage de Caux, voir Jacqueline Musset, «L'originalité de la coutume du pays de Caux en matière successorale", Recueil de l'association des amis du Vieux Havre, $\mathrm{n}^{\circ} 43,1986$, p. 1-18.

59. BNF, ms. 6570. 
que celles évoquées précédemment. Cependant, le principe de base de cette succession en Caux, un droit d'aînesse qualifié d'absolu réduisant à peu de choses la part des puînés, souleva la désapprobation de certains commentateurs, à l'image de Basnage, pour qui les articles 293 et 294 achevaient de rendre la condition des puînés «fort dure et fort fâcheuse ${ }^{60}$ ». Au-delà de ces critiques, Jacqueline Musset a montré que les critiques à l'égard des successions en Caux furent largement présentes dans les cahiers des bailliages secondaires concernés, et qu'elles exprimaient le rejet total du très fort droit d'aînesse régissant ces successions ${ }^{61}$. Ainsi, était dénoncée l'injustice de ce droit qualifié de "privilège barbare " réduisant les puînés "à la misère et même à la mendicité62 ». La plupart de ces cahiers ne se cantonnaient d'ailleurs pas à réclamer une réforme mais bel et bien la suppression de ce régime particulier ${ }^{63}$. Or, on le sait, l'influence des hommes de loi dans l'élaboration et la rédaction de ces cahiers fut prépondérante et leur contenu reflète sans doute davantage les sentiments des juristes que ceux de la population dans son ensemble ${ }^{64}$. À partir de là, il ne semble pas déraisonnable de penser qu'une vingtaine d'années avant la rédaction de ces cahiers le rejet du particularisme successoral en pays de Caux s'était développé chez les juristes normands et qu'il avait trouvé à s'exprimer dans ce projet de réforme de la coutume de Normandie.

60. Henri Basnage, op. cit., t. I, p. 462. Roupnel de Chenilly estimait quant à lui, dans sa note sous l'article 279: «Cette loi est déjà trop onéreusse au puîné, et on ne peut, sans injustice, rendre, pour l'agrément de l'aîné, sa condition plus mauvaise » (Pesnelle, op. cit., t. I, p. 319).

61. Jacqueline Musset, «Les sentiments des normands sur leur Coutume dans les cahiers de doléances de 1789 ", Annales de Normandie, $\mathrm{n}^{\circ}$ 39, 1989, p. 64.

62. Ibid., p. 66. Jacqueline Musset cite, en note, toute une série de protestations contenues dans ces cahiers et particulièrement révélatrices du ressentiment de la population comme celui de la paroisse de Saint-Jean-du-Cardonnay selon lequel " la coutume de Caux est tout à fait préjudiciable et contraire au droit naturel et légitime des enfants, en ce qu'elle dépouille les puînés du bien qui leur appartient pour le mettre sur la tête d'un aîné, lequel n'a souvent presque pas paru à la maison paternelle..."

63. Ibid., p. 67. Voir également, dans le Journal de Normandie du 18 mars 1789, la présentation d'une brochure contre la coutume de Caux rédigée par Pierre Ebran, avocat au parlement de Normandie et dans laquelle on pouvait lire: "avez-vous placé à la tête du cahier de vos doléances l'abus qui met entre vos aînés et vos puînés la même différence à peu près qu'entre des enfants légitimés et des bâtards; celui qui doit vous faire craindre de devenir père de nombreuse famille, et qui arrête nécessairement chez vous les progrès de la population; celui qui tend chez vous à semer, à entretenir, à perpétuer la désunion, et l'esprit de discorde et d'inimitié entre les frères et leurs descendants?"

64. Jean-Louis Halpérin, op. cit., p. 48. 
$\mathrm{Au}$ terme de ces développements, il est certain qu'en élaborant ses Observations qui devaient amener à une réforme de la coutume normande, l'auteur s'était attaché à consulter largement la doctrine et la jurisprudence, et qu'il s'était efforcé de répondre autant que faire se pouvait à leurs aspirations. Cependant, là n'était pas l'unique ni même le principal objectif de la réforme. Le chancelier Maupeou aspirait plus largement à l'unification du droit dans le royaume et à certains égards, les Observations sur la Coutume de Normandie apparaissent comme une première étape en ce sens.

\section{Une réforme portant les prémices d'une unification}

En envisageant de réformer les coutumes du royaume, le chancelier Maupeou avait pour finalité de les ramener toutes vers « une coutume mère ». Les modifications envisagées par les Observations sur la Coutume de Normandie s'attachèrent donc à ce travail et prévoyaient d'y parvenir par deux moyens, d'une part en utilisant le droit commun coutumier pour pallier les lacunes de la coutume de Normandie, d'autre part en retranchant de cette coutume certains particularismes.

\subsection{Le droit commun coutumier comme remède aux carences de la coutume de Normandie}

Afin de parvenir à une unification du droit coutumier du royaume, l'auteur des Observations sur la Coutume de Normandie allait s'appuyer sur les silences, les lacunes de cette coutume afin d'y insérer des dispositions permettant de donner corps à un droit commun coutumier.

Telle était son intention ouvertement affichée relativement au dernier titre de la coutume de Normandie consacré aux servitudes. Considérant ce dernier comme fortement lacunaire, il entendait combler ces manques en transposant purement et simplement dans ce titre les dispositions contenues dans le titre IX de la coutume de la prévôté et vicomté de Paris ${ }^{65}$.

Tout aussi explicites étaient ses propos quant au titre « Des choses censées meubles ». Sans revenir sur l'ensemble de ce titre, le projet de réforme s'attardait sur l'article 507 qui portait: «Les rentes constituées

65. «Dans ce titre il manque plusieurs dispositions sur différents cas; il sera à propos d'ajouter celles de la coutume de Paris sur ces différents cas» (BNF, ms. 6570). Les articles consacrés aux servitudes dans la coutume de Normandie, au nombre de 16, étaient effectivement moindres que dans la coutume de Paris puisque le titre qui leur était consacrées en comportait 36. 
à prix d'argent, encore qu'elles soient raquitables, sont réputées immeubles, et néanmoins si elles sont baillées en eschange contre héritage, ledit contrat est sujet à clameur lignagère ${ }^{66}$. " La volonté de l'auteur n'était pas de revenir sur cet article dans la mesure où ce dernier était en parfait accord avec la coutume de Paris et constituait selon Bourjon, le droit commun du royaume ${ }^{67}$. En revanche, il entendait profiter du mutisme du texte de la coutume normande sur le partage de ces rentes afin d'éclaircir la manière dont celui-ci se pratiquait dans cette province et pour y apporter des modifications dans le sens de l'unité.

En Normandie, l'usage était de partager les rentes constituées à prix d'argent selon les coutumes des lieux où les biens des débiteurs étaient situés. Selon Basnage, il s'agissait d'un usage ancien tirant son fondement de ce qu'autrefois on estimait que « la constitution de rente en deniers ne pouvoit valoir, si l'on y affectoit spécialement quelque fonds, ou qu'au moins celui qui s'obligeoit ne possédât quelques héritages ${ }^{68}$ ». Cet usage, confirmé par deux arrêts des 25 août 1546 et 4 juin $1603^{69}$, s'était depuis perpétué. Dans les autres provinces du royaume, le partage des rentes constituées se réglait suivant la coutume du domicile du créancier. Le fondement de cette pratique y était tout différent. On estimait que les rentes constituées suivaient la personne et que c'était dans la personne du créancier que résidaient de telles rentes ${ }^{70}$.

Selon l'auteur des Observations, cette règle avait l'avantage de la simplicité, au contraire de l'usage normand qui établissait un partage pour le moins complexe. À l'appui de cette assertion, il évoquait le cas d'un débiteur dont les biens, situés en Normandie, étaient partie en bourgage, partie hors bourgage, partie en Caux et partie hors de

66. Bourdot de Richebourg, op. cit., t. IV, p. 83.

67. «La coutume de Paris répute les rentes constituées immeubles; c’est sa disposition, et tel est le droit commun du Royaume» (François Bourjon, Le Droit commun de la France et la Coutume de Paris réduits en principes, Paris, Grangé, 1770, t. I, p. 329). Certaines coutumes cependant classaient ces rentes parmi les meubles comme celles de Reims et de Troyes; voir Jean Bart, op. cit., p. 215-216.

68. Henri Basnage, Euvres, op. cit., t. II, p. 513. Pesnelle notait à propos de cet usage: «La coutume semble plutôt considérer ces rentes par l'hypothèque qui est réelle et dépendante du fonds qui y est engagée que par l'obligation personnelle» (op. cit., t. II, p. 659).

69. Henri Basnage, Euvres, op. cit., t. I, p. 498.

70. François Bourjon, op. cit., t. I, p. 329. Voir encore Pesnelle: « En la coutume de Paris, les rentes constituées sont plutôt estimées par l'obligation personnelle, parce qu'elle est principale, que par l'hypothécaire qui n'est qu'accessoire. Elles se partagent entre les héritiers, suivant la coutume du domicile du créancier, en la personne duquel l'obligation est censée résider; puisque c'est lui qui peut exercer les actions qui en naissent, en cette même coutume» (op. cit., t. II, p. 659). 
Caux. Dans une telle hypothèse, une même rente devrait être partagée différemment selon la situation des biens ${ }^{71}$. Pour renforcer la nécessité de mettre un terme à cette pratique, il insistait encore sur la division des juristes normands quant à l'application de cette règle lorsque le créancier était normand et que le débiteur avait ses biens constitués en une autre province ${ }^{72}$. Basnage lui-même reconnaissait ces difficultés: "Mais quand une succession échet en cette Province, et qu'il se trouve des rentes dûes par des personnes dont les biens sont situez hors cette Province, comme ces rentes n'ont point d'assiette, on a eu de la peine à décider par quelle coutume le partage s'en devoit régler ${ }^{73} \ldots$ » Tout en constatant l'usage en vigueur dans la province, Roupnel de Chenilly, s'appuyant sur l'opinion de Le Brun qui critiquait la manière de partager ces rentes en Normandie ${ }^{74}$, émettait des réserves sur son bien-fondé. Il se ralliait finalement à la position de Froland, selon lequel l'usage normand était «fort dangereux, extraordinaire, incommode, et même exorbitant du Droit commun ${ }^{75}$ »; et il estimait que dans la succession d'un homme mort, domicilié en Normandie, on devait considérer une rente affectée sur des biens situés hors la province comme rente normande et la régler par la coutume de Normandie, nonobstant les préjugés contraires de Basnage $^{76}$.

71. BNF, ms. 6570. Pesnelle décrivait la même situation: "Ces rentes se partagent suivant la situation des biens obligés: de sorte que s'il y a des biens en Caux et hors de Caux, la rente se partage entre les héritiers du créancier, en partie comme si elle étoit un bien de Caux, et en partie comme si elle étoit un immeuble situé hors de Caux, par rapport et proportions à la valeur des biens que l'obligé possède en l'un et l'autre lieu» (op. cit., t. II, p. 659).

72. BNF, ms. 6570.

73. Henri Basnage, op. cit., t. II, p. 498.

74. «Le Brun, [...], trouve de l'inconvénient dans notre manière de régler les rentes constituées par la situation des biens qui y sont hypothéquées...» (Pesnelle, op. cit., p. 660, n. 1).

75. Louis Froland, Mémoires concernans la nature et la qualité des statuts, Paris, 1729, t. I, p. 467. Voir encore p. 599 et suiv.

76. Pesnelle, op. cit., t. II, p. 659. Dans sa note 7, sous l'article 329 de la coutume de Normandie, il relevait encore: «Notre jurisprudence, sur le partage des rentes constituées, est contraire au droit commun du royaume. » Et plus loin : « La province demanda au roi, dans ses cahiers de remontrances aux états de 1643, que les rentes constituées se partageassent suivant la coutume du domicile du créancier, pour ôter, disoit-elle, les occasions de discordes résultantes du partage relatif à la situation des biens du débiteur: cet article important a été négligé » (op. cit., t. I, p. 354). Jean-Baptiste Flaust, dont l'ouvrage parut après la rédaction de ces Observations sur la Coutume de Normandie, estimait quant à lui : «Cette jurisprudence est trop ancienne, et nous y sommes trop accoutumés pour tenter de s'élever au contraire»; et ajoutait : «Il est remarquable que cet usage et cette jurisprudence n'ont lieu que 
Fort de tout cela, l'auteur estimait donc absolument nécessaire de mettre un terme à cette pratique. Selon lui, rien ne s'opposait à cette modification dans la mesure où « la coutume de Normandie ne contient pas de loi contraire ${ }^{77}$ ", et où il ne s'agissait que de faire cesser une jurisprudence et d'établir une loi unique pour la province. Cette réforme serait donc nécessairement bénéfique puisqu'elle uniformiserait sur ce point le droit coutumier du royaume et ferait cesser les difficultés qui avaient émaillé, jusqu'alors, les partages de rente dans la province.

Afin de parvenir à la réalisation de cette entreprise d'unification du droit coutumier du royaume, les Observations sur la Coutume de Normandie n'entendaient pas cantonner leurs efforts aux seules lacunes du texte normand de 1583 . Il s'agissait également de mettre à bas certains particularismes normands qui constituaient d'indéniables obstacles au succès d'un tel projet.

\subsection{La nécessité de supprimer certains particularismes}

Robert Villers notait, lors de la publication de ces Observations sur la Coutume de Normandie, que ce projet restait somme toute modéré dans la mesure où il laissait subsister, sans y apporter de grandes modifications, les principaux traits caractéristiques de la coutume normande ${ }^{78}$. Ainsi, le régime successoral, notamment l'exclusion des filles, l'un des «caractères originaux » de la coutume de Normandie, demeurait dans son entier. De même, en matière de régime matrimonial, rien ne semblait devoir venir atténuer l'absence de communauté entre époux ${ }^{79}$. Au

dans le cas où les débiteurs sont domiciliés en Normandie, et où ils ont leurs biens dans cette province, parce que la coutume n'a point d'empire hors son ressort [...] il faut observer que nous tenons que la jurisprudence de Normandie, qui veut que les rentes constituées se partagent suivant la nature ou la situation des biens du débiteur, n'a son application, et n'est à suivre que quand deux choses concourent: savoir; quand le créancier est domicilié en Normandie, et quand le débiteur demeure et à ses biens dans la même province. Hors le cas de ce concours, les rentes constituées doivent suivre le domicile du créancier; c'est par la coutume du domicile du créancier, qu'on règle le partage des rentes constituées dans le droit commun et général. On en jugera de même en Normandie, quand le débiteur sera domicilié, ou quand il aura ses biens hors la province. Ce serait donc être dans l'erreur que de tenir indéfiniment qu'en Normandie les rentes ne suivent point le domicile du créancier, comme à Paris et ailleurs; toutes rentes hypothèques appartenant à des domiciliés en Normandie, sont censées de la coutume de Normandie» (op. cit., t. I, p. 284).

77. BNF, ms. 6570.

78. « Nullement révolutionnaire, il fait la part belle à la tradition...» (Robert Villers, op. cit., p. 359).

79. Sur les caractères originaux de la coutume de Normandie, voir Jean Yver, «Les caractères originaux de la coutume de Normandie», art. cité. 
contraire, comme le relevait Robert Villers, le projet de réforme paraissait vouloir pousser à l'extrême la logique de l'absence de communauté posée par l'article $389^{80}$ de la coutume de Normandie et ainsi supprimer du texte l'article 329 qui, selon l'auteur, y était en totale contradiction ${ }^{81}$.

En dépit de cette retenue apparente, ce projet n'en envisageait pas moins de revenir sur des aspects fondamentaux de la coutume de Normandie, notamment son caractère extrêmement lignager et sa protection du patrimoine familial ${ }^{82}$. Si cette orientation n'était pas exclusive à la coutume de cette province, les autres coutumes de l'ouest du royaume

80. Cet article était rédigé comme suit: "Les personnes conjointes par mariage ne sont communes en biens, soient meubles ou conquêts immeubles, ainsi les femmes n'y ont rien qu'après la mort du mari. » Concernant la rédaction de cet article, l'auteur des Observations préconisait d'en retrancher les derniers termes car dans les provinces où la communauté était admise, les femmes n'avaient rien sur les conquêts ni sur les meubles qu'après la mort du mari. Parmi les auteurs normands, Pesnelle avait été interpellé par cette rédaction puisqu'il indiquait à propos de cet article 389 : «La coutume en cet article se sert d'un mauvais argument, pour prouver que le mari et la femme ne sont point communs en biens, quand elle propose pour fondement de cette conclusion, que les femmes n'ont rien aux meubles ni aux immeubles, sinon après la mort du mari : car dans les coutumes, dans lesquelles les femmes sont reconnues être en communauté de biens avec leurs maris, il est certain qu'elles n'ont rien aux biens qui entrent en la communauté, sinon après la mort de leurs maris; étant indubitable, que tant que le mariage subsiste, le mari est le maitre de tous les biens de la communauté, dont il peut disposer indépendamment de sa femme, laquelle partant n'y a rien, sinon après la mort du mari » (op. cit., t. II, p. 446).

81. "Serait-il possible de laisser subsister dans une même coutume des dispositions aussi contradictoires? » (BNF, ms. 6570). Cet article de la coutume disposait : « La femme après la mort du mari a la moitié en propriété des conquêts faits en bourgage constant le mariage, et quant aux conquêts faits hors bourgage, la femme a la moitié en propriété au bailliage de Gisors, et en usufruit au bailliage de Caux, et le tiers par usufruit aux autres bailliages et vicomtez. » Il était reproché à cet article de faire que les héritiers de la femme prédécédée avaient la moitié en propriété sur tous les conquêts faits par le mari dans le comté de Gisors et sur les conquêts faits en bourgage dans tous les autres bailliages. Or la coutume de Normandie rejetant, par l'article 389, la communauté entre époux, il devait s'ensuivre que les héritiers de la femme prédécédée n'eussent rien sur les conquêts faits par le mari. Sur cette question de la communauté entre époux dans la coutume de Normandie, voir les développements de Virginie Lemonnier-Lesage, op. cit., p. 267-292.

82. Voir notamment Robert Besnier, La Coutume de Normandie. Histoire externe, Paris, Sirey, 1935, p. 16-17; consulter également Jean Yver, «Les caractères orignaux de la coutume de Normandie", art. cité, p. 340 et suiv. Jacqueline Musset indique à ce propos que les autres coutumes de l'ouest poursuivaient le même but mais que celle de Normandie allait plus loin en ce sens, ( «Le parlement et la coutume de Normandie», art. cité, p. 132). Dans son dictionnaire, David Hoüard indiquait sous le terme remplacement: «La conservation des biens dans la famille est le principal but auquel tendent presque toutes les dispositions de la coutume de Normandie » (op. cit., t. IV, p. 84). 
manifestant une même préoccupation, il s'avère qu'en Normandie ce souci était poussé à l'extrême ${ }^{83}$.

Le caractère lignager s'exprimait pleinement à travers l'article 245 de la coutume normande ${ }^{84}$. Suivant cet article, la règle paterna paternis materna maternis s'appliquait suivant le principe de côté et ligne. Dès lors, seuls les parents de la ligne d'où provenaient les biens étaient considérés comme héritiers. Par conséquent, dans l'hypothèse où les parents de cette ligne venaient à manquer, les propres étaient considérés comme vacants et attribués au seigneur qui les recueillait par droit de déshérence, plutôt qu'aux parents de l'autre ligne ${ }^{85}$. Selon Basnage, cet usage, qu'il qualifiait lui-même de dur, procédait de ce qu'autrefois les seigneurs ne concédaient les fiefs qu'à vie, qu'ils les donnèrent ensuite aux mâles, et enfin à la famille, "c'est-à-dire à ceux qui étoient $\mathrm{du}$ nom et ligne du premier possesseur, et par cette raison on les apeloit fiefs paternels, pour les reconnoitre et les distinguer d'avec les autres $^{86} »$. Incontestablement, la raison pour laquelle les Observations sur la Coutume de Normandie envisageaient de réformer cet article résidait dans le complet décalage existant entre ce dernier et l'évolution qu'avait connue le droit en la matière depuis le $\mathrm{xvI}^{\mathrm{e}}$ siècle $^{87}$. En effet, même si certaines coutumes de l'ouest avaient conservé une disposition similaire $^{88}$, nombreuses étaient celles qui s'étaient assouplies et qui

83. Jean Yver, Les Caractères originaux du groupe de coutumes de l'Ouest de la France, Paris, Sirey, 1952, p. 41 et 44; Jacqueline Musset, «Le parlement et la coutume de Normandie», art. cité, p. 132.

84. Article 245 : «Les héritages venus du côté paternel retournent toujours par succession aux parents paternels, comme aussi sont ceux du côté maternel aux maternels, sans que les biens d'un côté puisent succéder à l'autre, en quelque degré qu'ils soient parents, ains plûtôt les Seigneurs desquels lesdits biens sont tenus et mouvants, y succèdent. »

85. Jean Yver, «Les caractères originaux de la coutume de Normandie», art. cité, p. 337 ; Jacqueline Musset, «Le parlement... », art. cité, p. 133.

86. Henri Basnage, Euvres, op. cit., t. I, p. 385. L'auteur notait encore: «On ne peut attribuer qu'à cela cette loi si extraordinaire, qui donne les biens aux seigneurs dont ils sont tenus, au préjudice des parens "; et plus loin: "Il faut donc que quelque puissante raison ait porté nos réformateurs à établir une loi si dure, qui préfère les seigneurs et le fisc aux parents lorsqu'ils ne sont point de l'estoc de l'auteur ou de l'acquéreur des héritages : cela ne peut être fondé que sur cette loi des fiefs, qui faisoit succéder les seigneurs au défaut des lignagers de ceux à qui l'investiture avoit été accordée.»

87. Jean Yver note que « cette solution logique avait été largement pratiquée au Moyen Âge dans les diverses coutumes françaises" ("Les caractères originaux de la coutume de Normandie», art. cité, p. 337).

88. Tel était le cas des coutumes de Bretagne, d'Anjou ou encore du Maine (Jean Yver, Les Caractères originaux du groupe des coutumes de l'Ouest..., op. cit., p. 46). 
avaient préféré appeler les non-lignagers aux dépens du seigneur. Telle fut notamment l'évolution suivie par la coutume réformée de Paris qui dans son article 330 portait: «Et s'il n'y a aucuns héritiers du costé et ligne dont sont venus les héritages, ils appartiennent au plus prochain habile à succéder de l'autre costé et ligne, en quelque degré que ce soit $^{89}$.» Désormais, le droit commun coutumier s'établissait donc autour de cette solution considérée comme plus équitable envers ceux qui, bien que n'appartenant pas à la ligne d'où provenaient les biens, étaient susceptibles d'être très proches du défunt ${ }^{90}$.

Aussi bien, dans un souci de justice, le projet de réforme souhaitait aligner la coutume de Normandie sur la plupart des autres coutumes du royaume en l'amputant d'une disposition qui, aux dires de Pesnelle, n'était rien moins qu'absurde ${ }^{91}$ et qui finalement faisait prévaloir le droit du seigneur sur celui de la famille $e^{92}$.

Pour ce qui a trait à la protection du patrimoine familial, la coutume de Normandie faisait encore œuvre originale, poussant la protection des propres à son paroxysme. À travers la réserve héréditaire et le retrait lignager, toutes les coutumes ont montré qu'elles entendaient protéger les biens de famille. Cependant, comme le notait Jean Yver, aucune n'est allée aussi loin dans ce sens que la coutume de Normandie ${ }^{93}$. En effet, alors que dans la plupart des coutumes du royaume le de cujus avait la liberté de vendre de son vivant ses propres sans que les héritiers aux propres pussent s'en offusquer, le droit normand prévoyait qu'avant tout partage de succession, les propres aliénés seraient remplacés sur les acquêts et à défaut sur les meubles ${ }^{94}$. Dès lors, les héritiers aux conquêts étaient assujettis au remplacement des propres aliénés. Une telle disposition n'était pas contenue dans le texte même de la coutume de 1583 mais résultait d'une jurisprudence constante, établie par de nombreux arrêts ${ }^{95}$, et entérinée par l'article 107 de l'arrêt de règlement du parlement de Rouen de 1666, dit des Placités, qui avait vocation

89. Charles Bourdot de Richebourg, op. cit., t. III, p. 53.

90. Jacqueline Musset, «Le parlement...», art. cité, p. 133.

91. Pesnelle, op. cit., t. I, p. 261.

92. Jean Yver, «Les caractères originaux de la coutume de Normandie », art. cité, p. 336.

93. Jean Yver, "Un trait de la protection lignagère en Normandie: la subrogation des acquêts et des meubles aux propres aliénés, dans les rapports entre catégories d'héritiers ", Études d'histoire du droit privé offertes à Pierre Petot, Paris, LGDJ, 1959, p. 617-627.

94. Ibid., p. 618.

95. Henri Basnage, op. cit., t. II, p. 132. 
à compléter le texte de la coutume ${ }^{96}$. Cette jurisprudence était le fruit d'une interprétation extensive de l'article 408 de la coutume de Normandie ${ }^{97}$. Ce dernier, inséré dans le titre relatif au douaire, prévoyait uniquement, comme d'autres coutumes, le remplacement des propres aliénés pendant le mariage afin de prévenir les avantages que pourrait faire un mari à sa femme ${ }^{98}$. Une telle règle se trouvait notamment dans la coutume réformée de Paris ${ }^{99}$ mais, à la différence de la Normandie, elle n'avait fait l'objet d'aucune interprétation et était appliquée strictement.

Bien qu'isolée dans le droit coutumier du royaume, cette interprétation emportait l'adhésion et même l'admiration des commentateurs normands. Ainsi, Basnage considérait qu'il s'agissait «d'une des plus sage disposition de la coutume » car «c'est honorer en quelque sorte la mémoire de nos ancêtres, que de conserver avec soin les biens qu'ils nous ont laissez: toutes les personnes sages s'en font volontairement un devoir et une obligation, et les dissipateurs de leurs patrimoines ont toujours été des objets de mépris ${ }^{100} »$. Il allait même jusqu’à regretter la frilosité des

96. Article 107 : «Les propres aliénez doivent estre remplacez au profit des héritiers aux propres, et au marc la livre, sur tous les aquests immeubles; et à faute d'acquests, le remploy en sera fait sur les meubles» (Charles Bourdot de Richebourg, op. cit., t. IV, p. 158).

97. Basnage, tout en adhérant à cette interprétation, indiquait lui-même que le texte de l'article 408 ne reconnaissait pas ce principe expressément et que cela avait pu donner lieu à certains doutes lorsque la question avait été posée devant certains parlements (CEuvres, op. cit., t. II, p. 132). De même Pesnelle estimait : "Quoique cet article CCCCVIII semble n'avoir ordonné le remplacement des propres, que pour empêcher les avantages indirects que les maris peuvent faire à leurs femmes; il est nécessaire d'en étendre la disposition, et de dire que le remplacement des véritables propres, c'est-à-dire, qu'on a possédé à droit successif, doit être fait sur les acquêts et au défaut d'acquêts, sur les meubles: de sorte que les héritiers aux acquêts et aux meubles ne peuvent rien prétendre en leur succession, que le propre n'ait été remplacé au profit de ceux qui en sont héritiers » (op. cit., t. II, p. 508).

98. Article 408: «Les remplois de deniers provenus de la vente des propres ne sont censez conquests, sinon d'autant qu'il en est accreu au mary, outre ce qu'il en avoit lors des espousailles; comme aussi les acquisitions faites par le mary, ne sont réputées conquests, si pendant le mariage il a aliéné de son propre jusques à ce que ledit propre soit remplacé» (Charles Bourdot de Richebourg, op. cit., t. IV, p. 80).

99. Article 232: "Si durant le mariage est vendu aucun héritage ou rente propre appartenant à l'un ou l'autre des conjoints par mariage, ou si ladite rente est rachetée, le prix de vente, ou rachat, est repris sur les biens de la communauté, au profit de celuy auquel appartenoit l'héritage ou rente: encores qu'en vendant n'eust esté convenu de remploy, ou récompense : et qu'il n'y ait eu aucune déclaration sur ce faire» (Ibid., t. III, p. 46).

100. Henri Basnage, op. cit., t. II, p. 131. 
autres coutumes en la matière comme le montrent ses propos à l'égard de l'article 232 de la coutume réformée de Paris:

Ce remploi introduit par la Coûtume et par les arrêts du parlement de Paris, n'a pas retranché tous les abus, ni donné remède à tous les inconvéniens qui naissent de l'aliénation des propres; on a point ateint le but et la fin principale que l'on devoit se proposer, à savoir la conservation des biens dans les familles. Tout l'éfet de ce remploi se termine à empêcher que les conjoints par mariage ne profitent point des biens l'un de l'autre; il n'a lieu que quand il s'agit du droit de communauté, et non du droit de succéder [...] Et quand un homme auroit aliéné tous ses propres, l'héritier aux propres n'en auroit aucune récompense, l'action de remploi étant inconnuë entre divers héritiers ${ }^{101}$.

En dépit de l'attachement des juristes normands à cette règle, il est certain, une nouvelle fois, qu'elle n'allait pas dans le sens du droit commun coutumier. En effet, ce dernier tendait dans son ensemble à affaiblir la protection des propres et à considérer comme droit commun la succession aux meubles et acquêts ${ }^{102}$. Dès lors, il n'est pas étonnant que l'auteur des Observations sur la Coutume de Normandie ait envisagé de réformer cette pratique en la dénonçant avec force. Afin de démontrer la nécessité de supprimer cet article 107 du règlement de 1666, il développait toute une série d'arguments.

Ainsi, il s'en prenait à l'autorité de l'acte qui avait imposé cette règle du remplacement. En effet, celle-ci ne s'intégrant pas dans le texte de la coutume de 1583 mais prenant place dans le règlement du parlement de Rouen de 1666, l'auteur se refusait à lui reconnaître une quelconque autorité. Selon lui, n'ayant pas été validé par des lettres patentes, cet arrêt de règlement ne pouvait être regardé comme ayant force de loi ${ }^{103}$. S'il admettait que cette cour souveraine pût interpréter la coutume, en revanche il lui déniait le pouvoir de « faire des loix nouvelles» contraires à la coutume et qui à ses yeux dépassaient la simple interprétation. Par cette attaque était ouvertement proclamée la volonté du pouvoir royal d'être seule source de droit, même en matière de droit privé. Cette

101. Ibid., p. 131-132.

102. Jean Yver, «Les caractères originaux de la coutume de Normandie », art. cité, p. 344-345.

103. "Ce règlement de 1666 n'a jamais acquis la force de loi, puisqu'il n'a point été confirmé par des lettres patentes» (BNF, ms. 6570). Sur ce point, Jacqueline Musset note cependant que sur les prières du parlement de Rouen : "Le roi accepta, en l'homologuant, de donner à ce document d'origine spontanée de l'une de ses cours supérieures de justice, la valeur attachée aux seules ordonnances émanant de lui-même et découlant de son pouvoir souverain de légiférer» («Le parlement et la coutume de Normandie», art. cité, p. 138). 
unité législative étant en effet la condition essentielle de la réussite de l'entreprise d'unification du droit. Au-delà du seul droit privé, la remise en cause de l'autorité des actes du parlement rouennais est à rapprocher du contexte politique dans lequel ces Observations sur la Coutumes de Normandie prennent place. Rédigées autour de l'année 1770, elles s'inscrivaient dans un climat politique des plus tendus. Le pouvoir royal et les cours souveraines du royaume étaient en lutte ouverte depuis une vingtaine d'année. Or en menant une politique de blocage systématique, par l'usage outrancier qu'ils faisaient de leur droit de remontrance, les parlements outrepassaient leur mission et s'immisçaient dans le champ du pouvoir législatif qui n'était pas le leur ${ }^{104}$. Dès lors, les diverses allusions faites dans les Observations au "souverain seul législateur» étaient sans aucun doute destinées à insister sur le rôle strict qui devait être le leur, et donc à condamner cette immixtion dans le pouvoir législatif du monarque.

Quant au contenu de cette règle, les Observations dénonçaient l'injustice manifeste de cet article 107 à l'égard des héritiers aux meubles et aux acquêts. Ces derniers étant bien souvent de plus proches parents que les héritiers aux propres, il était tout à fait incompréhensible qu'ils fussent lésés par une telle disposition ${ }^{105}$. Un autre grief avancé était celui de l'absence de fondement de cette règle. Par là, l'auteur s'en prenait à l'interprétation extensive de l'article 408 pratiquée par les juristes normands et insistait sur le fait qu'il n'y avait dans le texte de la coutume de Normandie « aucune disposition qui tende à admettre ces prétendus propres par subrogation ${ }^{106} »$. Il reprochait encore avec vigueur la large acception qui était donnée au terme aliénation, car celle-ci s'entendait même « dans des cas où la sortie du bien hors du patrimoine propre n'a entraîné aucun accroissement corrélatif des meubles et acquêts ${ }^{107}$ ». Cela dépassait donc les termes mêmes de l'article 107 et aboutissait non plus

104. François Saint-Bonnet et Yves Sassier, Histoire des institutions avant 1789, Paris, Montchrestien, 2011, p. 332.

105. BNF, ms. 6570 : «Cette jurisprudence [...] est entièrement défavorable à l'héritier aux meubles et acquêts, qui cependant comme lignager le plus proche, semblerait mériter plus de faveur qu'un héritier aux propres qui peut être un parent fort éloigné et jusques dans le sixième et le septième degré. " Il est à noter que cet argument était rejeté par Basnage, op. cit., t. II, p. 132.

106. BNF, ms. 6570.

107. Jean Yver, "Un trait de la protection lignagère en Normandie... », art. cité, p. 621-622. L'auteur des Observations s'en prenait notamment à des arrêts qui avaient ordonné le remplacement sur les acquêts des propres qu'un homme avait vendus en dépit du fait que ce dernier avait utilisé le prix de la vente pour rembourser des rentes qui grevaient d'autres de ses propres. 
à conserver les anciens propres mais à les augmenter. Enfin, il mettait en avant l'isolement du droit normand en insistant sur le fait qu'aucune des coutumes du royaume, dans lesquelles on trouvait un article similaire à l'article 408, n'était allée dans le même sens que lui et qu'elles avaient toutes adhéré «à la maxime constante, que les biens des successions doivent se partager dans l'état où ils se trouvent ${ }^{108}$ ».

Si l'on s'en tient, au terme de cette étude, au but ultime poursuivi par Maupeou qui était de parvenir à l'unification du droit coutumier du royaume, le projet de réforme de la coutume de Normandie, présenté par l'auteur des Observations, peut paraître bien en deçà des ambitions du chancelier. En effet, comme l'écrivait Robert Villers, cette réforme n'était «nullement révolutionnaire ${ }^{109} »$. Ainsi, sur les vingt-quatre titres que contenait la coutume de Normandie, moins de la moitié firent l'objet de modifications. Surtout, ce projet laissa subsister les principales caractéristiques de la coutume normande qui en faisaient sa particularité et symbolisaient «son archaïsme», notamment l'exclusion successorale des filles et le maintien de l'absence de communauté entre époux.

Pour autant, il est indéniable que ce travail était empreint d'un réalisme certain dans un contexte politique pour le moins hostile. La constance avec laquelle il s'évertua à répondre aux aspirations des juristes normands attachés, on le sait, à leur coutume, pouvait laisser envisager sinon un accueil favorable du moins une résistance atténuée de leur part. Mais, surtout, si l'on se rappelle la démarche de Maupeou qui visait dans une première étape "à réduire à leurs différences et à leurs exceptions » les coutumes du royaume, à discuter, déterminer d'une manière précise et à soumettre à l'expression la plus simple, la plus vulgaire et la moins équivoque ces différentes coutumes, il est incontestable que les Observations sur la Coutume de Normandie répondaient parfaitement à cette première phase dans la mesure où on y retrouvait l'effort de simplification demandé et qu'étaient conservées les principales spécificités normandes. Dès lors, il semble qu'il faille davantage appréhender ces observations comme un premier travail, une étape, vers la voie de l'unification plutôt que comme un projet de réforme définitivement abouti de la coutume de Normandie.

Docteur en histoire du droit

108. BNF, ms. 6570.

109. Robert Villers, op. cit., p. 358. 